\title{
Tissue factor expression in normal and abnormal mammary gland
}

To the editor - In the February 1996 issue of Nature Medicine, Contrino et al.' report that tissue factor (TF) can be localized by immunohistochemical methods in tumor cells and in the endothelial cells lining tumor-associated vessels in breast cancer (cases $n=7$ ) but not in those of benign, fibrocystic disease of the breast ( $n=10)$, using antibodies generated in our laboratory ${ }^{2,3}$. The authors conclude that TF is a marker of the initiation of angiogenesis in human breast cancer. Although we have also suggested that expression of TF has an important role in angiogenesis ${ }^{4}$, we were unable to confirm the simple relationship between tumor cell expression of TF and specificity for the malignant phenotype in breast cancer. In contrast to Contrino et al., in proliferating disorders of the human mammary gland like fibrocystic disease or in fibroadenomas, we observed expression of $\mathrm{TF}$ in both myoepithelia and luminal epithelias. Also, we demonstrated robust expression of TF in pregnancy-stimulated breast, which was underlined by the finding of detectable amounts of TF antigen in normal human mother milk (112.1 $\pm 74.5 \mathrm{ng} / \mathrm{ml}, n=9)$

Immunohistological detection of tissue factor (TF) antigen in representative benign ( $a$ ) and malignant $(b, c)$ lesions of the mammary gland (immunoperoxidase procedure). Paraffin sections (4 $\mu \mathrm{m}$ thick), processed as previously described ${ }^{3}$, reacted with monoclonal antibody to human TF. $a$, fibrocystic disease with lobular secretion. Note the strong TF staining in activated epithelia (E) $\times 190$. $\quad b$ and $c$, Invasive ductal breast carcinomas with different TF antigen expression in tumor cells $(T) \times 190$. In $b$, note the strong TF staining in tumor cells of a carcinoma with high TF content of the tumor tissue extract $(50.4 \mathrm{ng}$ $\mathrm{TF} / \mathrm{mg}$ protein). In c, weak and inhomogeneous expression of TF in a carcinoma with low TF antigen content ( $1.2 \mathrm{ng} \mathrm{TF} / \mathrm{mg}$ protein). The specificity of the immunohistochemical staining was confirmed by competitive inhibition of the anti-TF monoclonal antibody binding using rTF (data not shown). using ELISA. Immunohistochemical studies on snap frozen $(n=115)$ and paraffin-embedded $(n=190)$ primary breast cancer tissues, using various monoclonal antibodies directed to human $\mathrm{TF}$, confirmed TF staining in an inhomogenous manner in terms of intensity and number of positive cells. A tendency was observed for more positive and stronger stained cells to be found in welldifferentiated structures of the tumors.

When we determined the TF antigen content using an ELISA, we found that the TF concentration per milligram protein of the tumor tissue extracts varied and was dependent on both the histologic grade and the histologic type of the breast cancer. Although expression of TF seems to correlate with both the tumor stage (colon cancer, unpublished observation) and the degree of histologic differentiation (pancreatic cancer ${ }^{6}$ ) in carcinomas other than breast cancer, we actually found a slightly negative correlation between histologic grade (BR-Grading) and TF antigen content ( $n=$ $360, P<0.05)$. On the other hand, in the identical carcinoma tissue extracts the urokinase (UPA) and urokinase-receptor

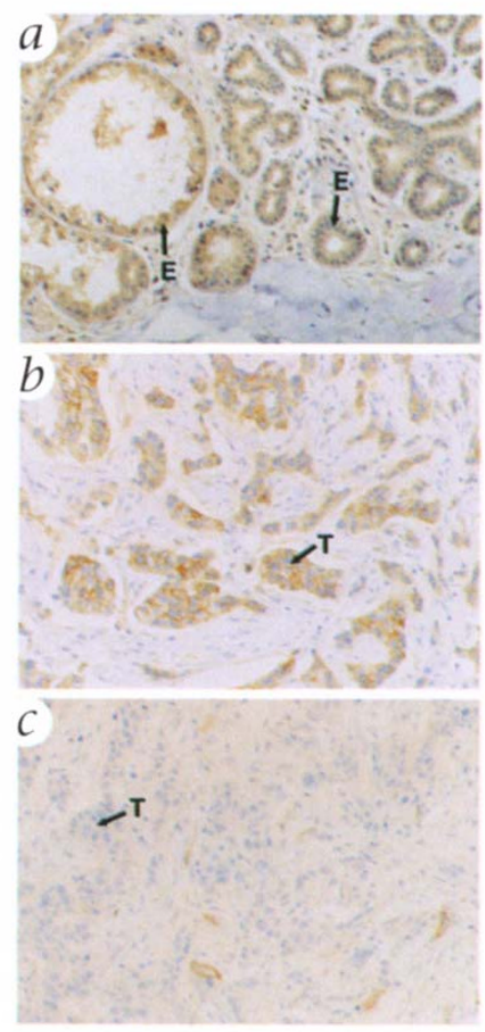

(UPAR) antigen levels, which are described as being correlated to invasive tumor growth, microvessel density and poor prognosis ${ }^{7-9}$, were positively correlated to the BR grading (UPA: $P<0.02$, UPAR: $P<0.001$ ). In agreement with Contrino et al., we suggest that the TF detected in human breast cancer is mainly functional, because the factor VII antigen content in carcinoma tissue extracts was positively correlated to its TF content ( $P$ $<0.002$ ).

Our immunohistochemical data show clearly that epithelial TF expression does not discriminate between benign and malignant growth in the mammary gland (see figure). Whether, in spite of this, TF expression by endothelial cells in the blood vessels of breast carcinomas is a useful marker that can distinguish between microvessels in benign and malignant lesions as described by Contrino et al., has to be confirmed by further more extended studies.

ThOMAS LUTHER, CONRAD FlÖSSEL, Sybille Albrecht, Mathias Kotzsch \& MarTin MÜLleR

Institute of Pathology

Technical University of Dresden

Fetscherstr. 74

01307 Dresden, Germany

Contrino et al. reply - We thank Luther and colleagues for their letter, in which they provide support for our findings of functional tissue factor (TF) in situ in invasive breast cancer ${ }^{1}$. Although the authors should be encouraged to provide additional information critical to understanding the spectrum of differences between their findings and our own, we have little quarrel with their general observations regarding the expression of $\mathrm{TF}$ in the myo-epithelial component of some benign breast tumors. Indeed, we suggested that a much larger study was needed to confirm our findings in benign breast disease. We commented that such a study might reveal a spectrum of staining for TF between little or none in completely benign disease, such as the tissue we studied (in the absence of a significant inflammatory component), to impressive staining for TF in so-called "normal" tissue adjacent to invasive, malignant disease, which we have observed but not published.

In contrast to Albrecht et al., however, we were able to confirm both positive and negative staining obtained with TF 